\title{
Thoracic ultrasound and SARS-COVID-19: a pictorial essay
}

\author{
Soccorsa Sofia ${ }^{1} \cdot$ Andrea Boccatonda $^{2} \cdot$ Marco Montanari $^{3} \cdot$ Michele Spampinato $^{1} \cdot$ Damiano D'ardes $^{2}$. \\ Giulio Cocco $^{2} \cdot$ Esterita Accogli $^{4} \cdot$ Francesco Cipollone $^{5} \cdot$ Cosima Schiavone $^{2}$
}

Received: 24 March 2020 / Accepted: 30 March 2020 / Published online: 16 April 2020

(c) Società Italiana di Ultrasonologia in Medicina e Biologia (SIUMB) 2020

\begin{abstract}
Thoracic ultrasound seems to adapt to the screening for lung involvement of patients with suspected or ascertained SARSCOVID-19 infection due to its characteristics of easy applicability. It can be also a relevant method in monitoring patients. B lines are early finding of COVID-19, even in mild-symptomatic subjects; in the most serious cases such as pre-ARDS or ARDS, the B lines end up filling the ultrasound image almost completely, until it merges, so as to create a single hyperechoic image named as "white lung", with distortion and irregularity of the pleural line. In advanced stage, lung consolidations are present, representing pulmonary pathological areas that are no longer normally ventilated.
\end{abstract}

Keyword Lung $\cdot$ COVID-19 $\cdot$ Virus $\cdot$ Ultrasound

A novel Coronavirus (2019-nCoV) was identified in hospitalized patients in Wuhan, China, in December 2019 and January 2020 [1]. Coronaviruses are enveloped, non-segmented positive-sense RNA viruses belonging to the family Coronaviridae and the order Nidovirales. They are broadly distributed in humans and other mammals [2]. The number of patients affected by COVID-19 is rapidly increasing almost all over the world.

The following procedure has been suggested for diagnosis of patients with suspected infection: performing real-time fluorescence (RT-PCR) to detect the positive nucleic acid of SARS-CoV-2 in sputum, throat swabs, and secretions of the lower respiratory tract samples [3].

Andrea Boccatonda

andrea.boccatonda@gmail.com

1 Emergency Department, Ospedale Maggiore AUSL Bologna, Bologna, Italy

2 Internistic Ultrasound Unit, SS Annunziata Hospital, "G. d’Annunzio" University, via dei Vestini, 66100 Chieti, Italy

3 Emergency Department, Infermi Hospital, Rimini, Italy

4 Department of Internal Medicine, Centre of Research and Learning in Ultrasound, Maggiore Hospital, Bologna, Italy

5 Clinica Medica Division and European Center of Excellence on Atherosclerosis, Hypertension and Dyslipidemia "SS. Annunziata" Hospital, Chieti, Italy
Preliminary data have shown that once infected, patients display an extremely variable clinical course, from mild symptoms (fever and cough) to bilateral interstitial pneumonia. In the most serious cases, infection progresses into acute respiratory distress syndrome (ARDS) with diffuse alveolar consolidations (diffuse patchy-like lesions) [4]. Furthermore, recent reports have classified patients into different clinical phenotypes from 1 to 5, with increasing clinical severity up to pre-ARDS (phenotype 4) and ARDS (phenotype 5).

Symptoms are very not specific, especially at an early stage, since they are common to most upper and lower airway diseases. The specific etiological diagnosis is performed through laboratory methods, but the main concern is to direct the patient to the best care setting (home observation, medical department/infectious disease clinic, sub-intensive ward or ICU).

Imaging methods are employed to assess the degree of lung involvement. The typical computed tomography (CT) feature of a patient with acute COVID-19 infection is that of ground-glass opacities (GGO) or mixed GGO, consolidation and vascular enlargement $[5,6]$; lesions are more likely to display a peripheral distribution and bilateral involvement, and to be lower lung predominant $[5,6]$. Although chest CT seems to be the method of choice, it is burdened by the risk of radiation exposure and concerns related to infection risk and the transport of the critical patient.

Thoracic ultrasound is an easy-to-learn and reliable method [7-13]. No advanced machinery or software is 
required. In an emergency setting or at bedside, the use of pocket-size ultrasound seems to be optimal. It is essential for the clinician to know and implement personal safety procedures before starting the examination. In particular, the physician performing the ultrasound must wear appropriate PPE. In case of a COVID-19 + patient with respiratory symptoms, these items are a surgical mask, gloves, a waterproof gown, and goggles or a visor; in the case of high risk of exposure to aerosols (patients subjected to continuous positive airway pressure or non-invasive ventilation), the use of a FFP2/3 mask, a double pair of gloves, goggles or a visor and boots is recommended. Ultrasound machine and probes must be disinfected with alcohol-based disinfectants before and after ultrasound, and thus equipped with disposable protective materials (probe cover).

The probe can be used as a stethoscope to auscultate/scan any portion of the lung not covered by bones. In practice, there are ultrasound scan protocols in emergency settings that provide for a minimum of $6-8$ scans per hemithorax on a patient on supine position ( $2 / 4$ anterior, 2 lateral and 2 posterior) $[8,12,13]$. One of the main limitations of thoracic ultrasound is that it cannot be used to examine the deep fields of the lung due to air interface and artifacts, but the physiopathology of COVID-19 disease provides for a predominant involvement of the pulmonary periphery [5, 6], thus favoring the application of the method.

The main ultrasound finding in COVID-19 pneumonia is that of an acute interstitial disease, which is represented by vertical hyperechoic artifacts that depart from the pleural line directed in depth. These are called B lines [14, 15]. If $\leq 3 \mathrm{~B}$ lines on a scan are physiological, especially in the middle-basal fields, the presence of a greater number is pathological, and the number of B lines correlates with disease severity (Figs. 1, 2, 3).

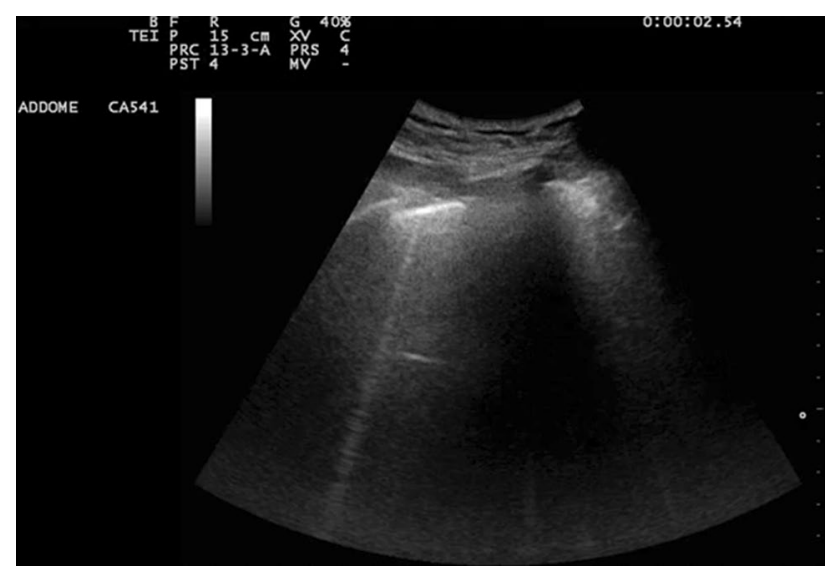

Fig. 1 Lateral chest scan. Single vertical hyperechoic artifact originating from the pleural line, called "B line"

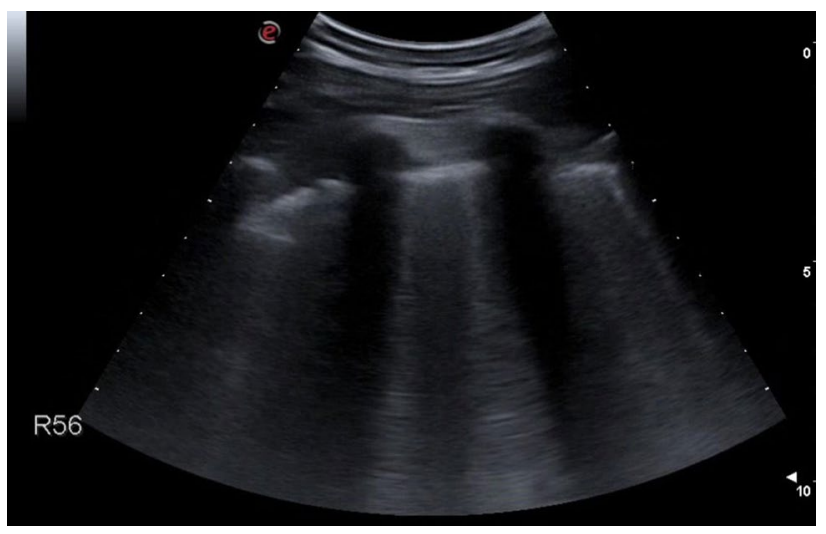

Fig. 2 Posterior chest area scan. Vertical artifacts ("B lines"), isolated and non-confluent
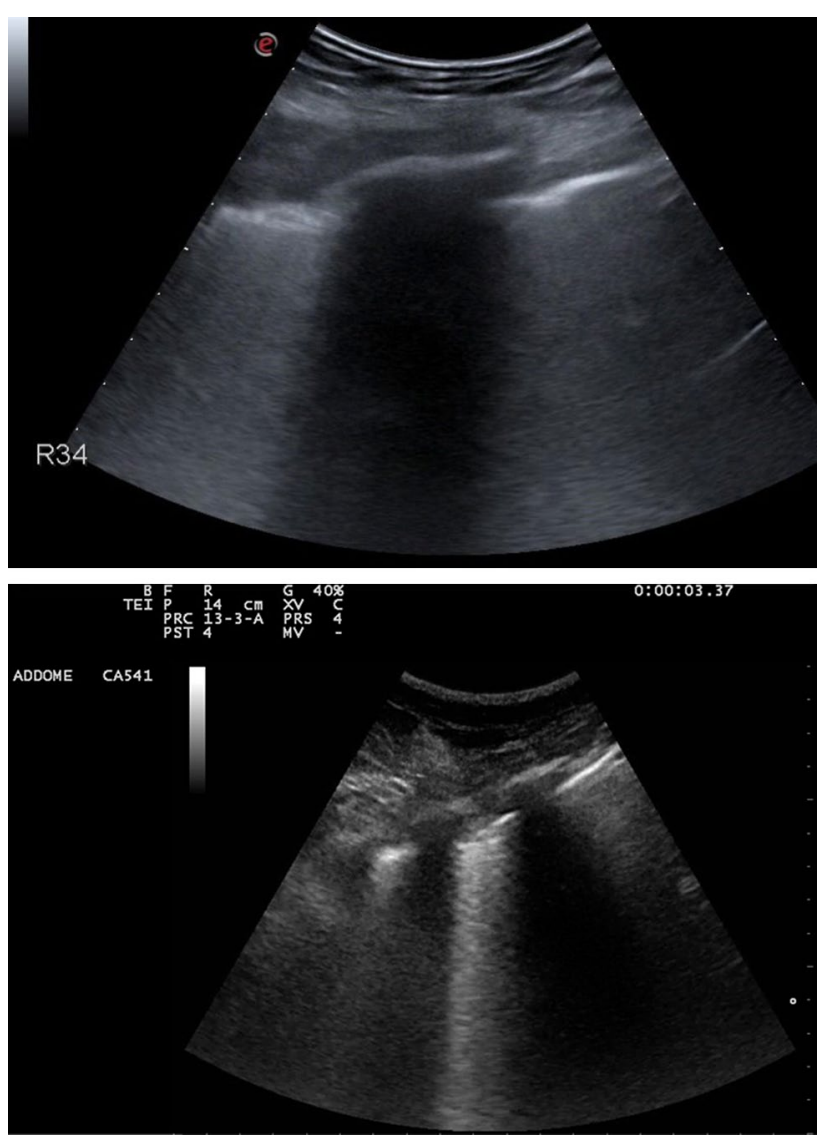

Fig. 3-4 Lateral chest scans. Vertical artifacts ("B lines"), numerous and coalescent at a stretch of pleural line of increased thickness

Indeed, the presence of some B lines spread over several scans seems to be an early finding of COVID-19, even in mildly symptomatic subjects; in the most serious cases such as those that are pre-ARDS or ARDS, the B lines end up filling the ultrasound image almost completely, until they 


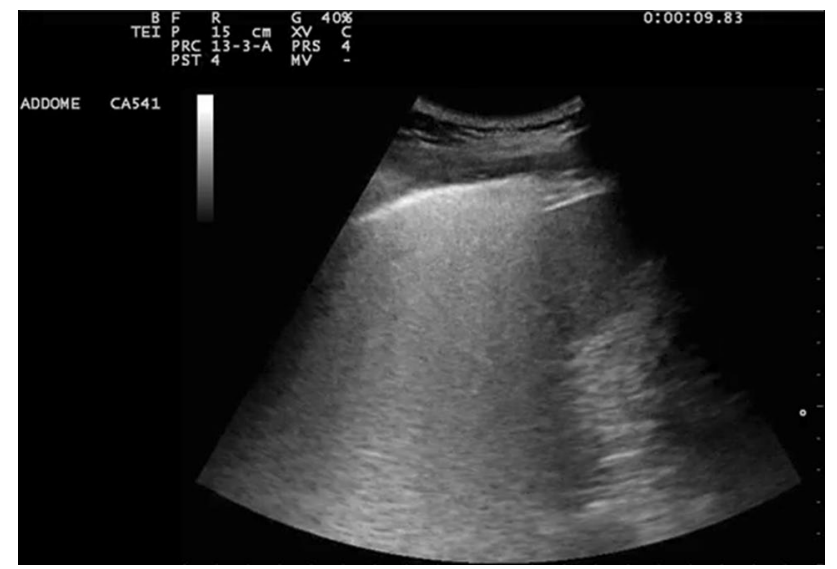

Fig. 5 Postero-basal chest scan. Lung field is occupied by numerous $\mathrm{B}$ lines that come together to form a single hyperechoic image. This aspect is named "white lung," referring to the ground-glass opacities found in the computed chest tomography

merge, creating a single hyperechoic image identified as "white lung" and distortion and irregularity of the pleural line (Figs. 4, 5).

Initially, those ultrasound findings were related to cardiogenic pulmonary edema, in which the B lines are present bilaterally, with a base-apex gradient and with progressive involvement depending on the severity of the disease [7]. Moreover, B lines are also found in other diseases such as bruises, chronic fibrotic lung diseases and ARDS. ARDS can be distinguished from cardiogenic pulmonary edema by the presence of multifocal and diffuse lung alterations, without a base-apex gradient, while chronic interstitial diseases differ by an extreme irregularity of the pleural line, which can be thickened and distorted, with the presence of focal subpleural interruptions (consolidations), and by the presence of uneven and irregular vertical artifacts (B lines) [7, 16, 17].

The difference between the features of $B$ lines found in cardiogenic interstitial edema and chronic fibrosing pathologies has recently led to new proposals for reclassification of the types of artifacts $[18,19]$. The ultrasound pattern of COVID-19 pneumonia seems to be very similar to that found in patients suffering from chronic pulmonary fibrosis; both are based on a prevalent interstitial involvement. In the most severe and advanced forms, lung consolidations are present, representing pulmonary pathological areas that are no longer normally ventilated (Figs. 6, 7, 8, 9).

In the early stage of COVID-19, lung changes are more localized, and mainly detected in the subpleural regions of one or both lungs (corresponding to patchy or segmental GGOs) (Figs. 2, 4). Later, viral infection and inflammatory response involve multiple lobes, leading to air loss and consolidations of some lesions surrounded by several B lines (Figs. 6, 7, 8). In more advanced and critical cases, ultrasound findings resemble other pneumonia features, such as

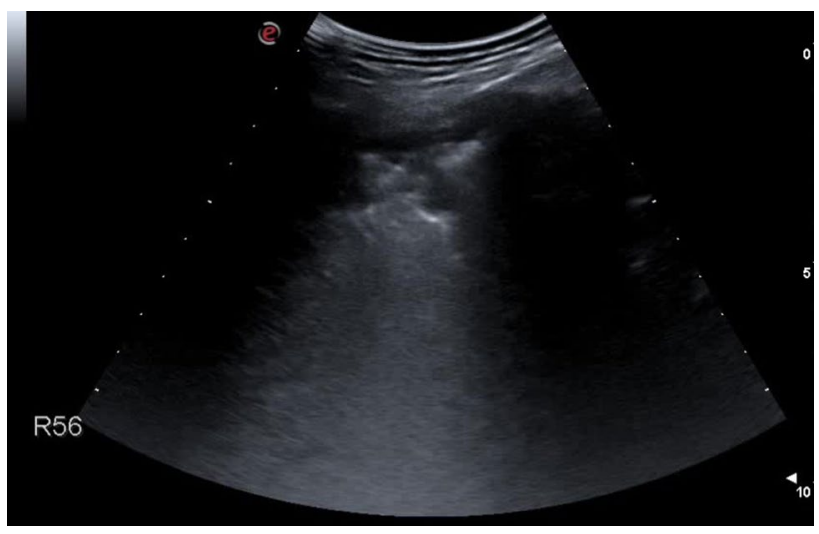

Fig. 6 Longitudinal posterior chest scan. Coarse subpleural thickening with very irregular margins

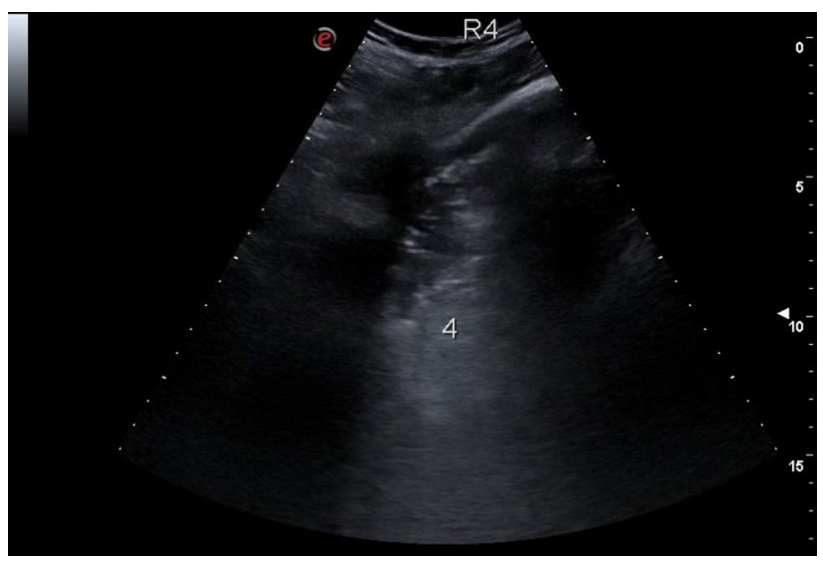

Fig. 7 Lateral chest scan. Coarse supleural thickening with thin aerial bronchograms

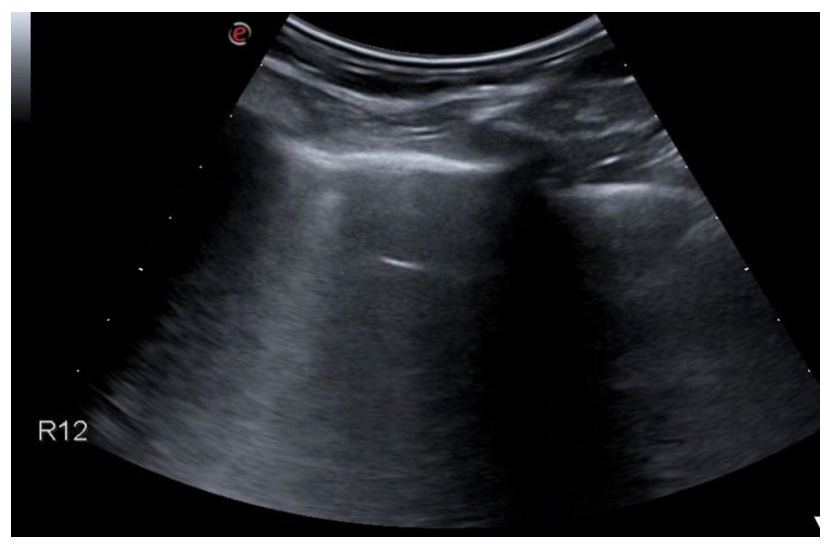

Fig. 8 Anterior chest longitudinal scan. Minute subpleural parenchymal thickening 


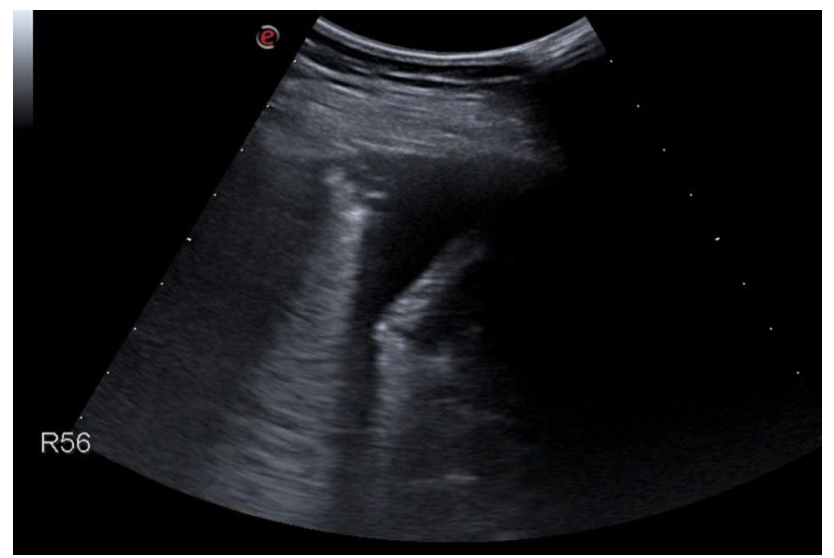

Fig. 9 Posterior chest longitudinal scan. Small basal pleural effusion

diffuse consolidations in both lungs, multiple and diffuse B lines (white lung), and occasionally by small amounts of pleural effusion (Fig. 5; Table 1) [20].

Therefore, thoracic ultrasound seems suitable for screening for lung involvement of patients with suspected or ascertained SARS-COVID-19 infection due to its easy applicability. It can be also a relevant method for monitoring patients. Indeed, follow-up of the previously described ultrasound findings according to the pre-established scan scheme can document improvements in or the worsening of the disease. It can thus, together with clinical-laboratory data, prompt therapeutic modifications. In an intensive care unit (ICU) setting, ultrasound can be a support for lung recruitment maneuvers, by assisting clinicians in ascertaining the possible re-expansion of the atelectasis areas (from consolidation to aerial artifacts).

Furthermore, the ultrasound evaluation of diaphragm excursion can provide relevant information about the patient's ventilatory capacity and, indirectly, about lung compliance [17, 21-23]. In this context, an ultrasound follow-up of the degree of diaphragmatic excursion could identify a worsening of the pneumonia requiring non-invasive or invasive ventilatory support, or on the contrary, an improvement, prompting a subsequent attempt to wean the patient off or downgrade the respiratory support (Fig. 10).

In conclusion, thoracic ultrasound can be used as a screening method for lung involvement in any care setting, even at the patient's home. The pathological characteristics (B lines and consolidations) revealed by ultrasound and their extent correlate with the severity of the disease, and can be employed as a monitoring method. Ultrasound is a useful tool to support the ventilated patient in terms of assessing diaphragm mobility and supporting recruitment maneuvers.

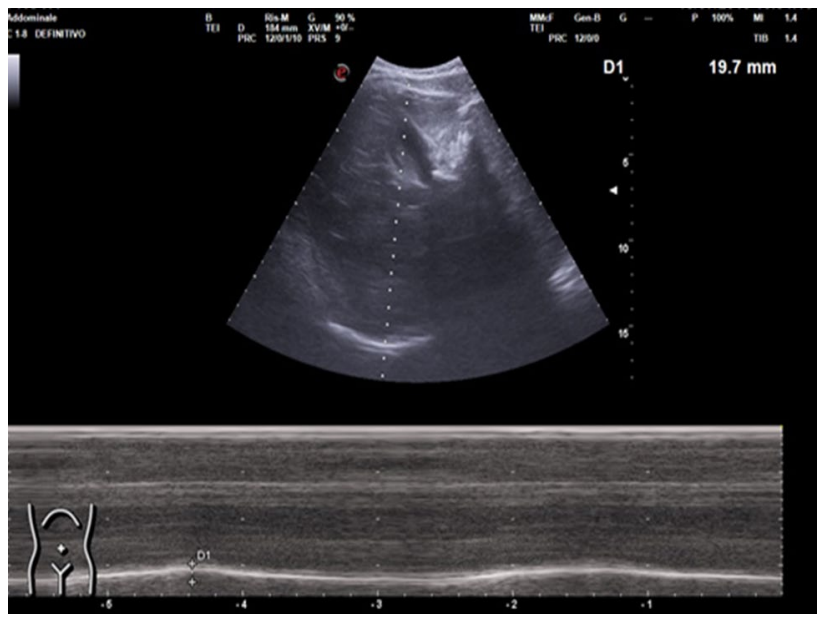

Fig. 10 Ultrasound evaluation of diaphragm excursion. An oblique subcostal scan should be performed by convex probe (scan for the gallbladder), by adjusting the depth of the image so as to completely highlight the liver margins, with the diaphragm represented as a hyperechoic line surrounding the liver. The M-mode option is activated by centering the line on the bed of the gallbladder and evaluating the degree of excursion of the diaphragm

Table 1 Suggested clinical and sonographic classification of COVID-19 pneumonia

\begin{tabular}{lll}
\hline Clinical stage & Clinical findings & Ultrasound findings \\
\hline I stage: simple disease & Patients with viral upper respiratory tract infection not complicated & Normal ultrasound pattern: A lines \\
II stage: mild pneumonia & Patient with pneumonia and no signs of severe pneumonia & Focal B lines \\
III stage: severe pneumonia & Fever or suspected respiratory infection, and at least one of the follow- & Diffuse and multifocal B lines \\
& ing: respiratory rate $>30$ acts/min, severe breathing difficulties or & Initial subpleuric consolidations \\
& SpO $<90 \%$ in ambient air & \\
IV stage: acute respiratory dis- & Respiratory failure that is not related to heart failure or overloaded & Diffuse and multifocal B lines \\
tress syndrome (ARDS) & with liquids. $200 \mathrm{mmHg}<\mathrm{PaO}_{2} / \mathrm{FiO}_{2} \leq 300 \mathrm{mmHg}$ (with PEEP or & Lung consolidations \\
& CPAP $\geq 5 \mathrm{cmH}_{2} \mathrm{O}$ or not ventilated) & White lung pattern \\
\hline
\end{tabular}

Adapted from https://www.siaarti.it/SiteAssets/News/COVID19\%20-\%20documenti\%20SIAARTI/Percorso\%20COVID-19\%20-\%20Sezione\%20 $1 \% 20 \% 20-\% 20$ Procedura\%20Area\%20Critica.pdf 


\section{Compliance with ethical standards}

Conflict of interest The authors declare no conflicts of interest and no funding sources.

Informed consent Informed consent to publish figures was obtained from patients.

\section{References}

1. Zhu N (2020) A novel coronavirus from patients with pneumonia in China, 2019. N Engl J Med 382(8):727-733. https://doi. org/10.1056/NEJMoa2001017Epub 2020 Jan 24

2. Huang C (2020) Clinical features of patients infected with 2019 novel coronavirus in Wuhan. China Lancet 395(10223):497-506. https://doi.org/10.1016/S0140-6736(20)30183-5Epub 2020 Jan 24

3. National Health Commission of People's Republic of China (2020) Pneumonia diagnosis and treatment of 2019-nCoV infection from Chinese NHC and CDC 2020. https://www.nhc.gov. cn/xcs/zhengcwj/202001/4294563ed35b43209b31739bd0785e 67/files/7a9309111267475a99d4306962c8bf78.pdf. Accessed 1 Feb 2020

4. Weiss P, Murdoch DR (2020) Clinical course and mortality risk of severe COVID-19. Lancet. https://doi.org/10.1016/S0140 $-6736(20) 30633-4$

5. Shi H, Han X, Jiang N, Cao Y, Alwalid O, Gu J et al (2020) Radiological findings from 81 patients with COVID-19 pneumonia in Wuhan, China: a descriptive study. Lancet Infect Dis. https://doi. org/10.1016/S1473-3099(20)30086-4

6. Pan F, Ye T, Sun P, Gui S, Liang B, Li L et al (2020) Time course of lung changes on chest CT during recovery from 2019 novel coronavirus (COVID-19) pneumonia. Radiology 13:200370. https ://doi.org/10.1148/radiol.2020200370

7. Soldati G, Demi M (2017) The use of lung ultrasound images for the differential diagnosis of pulmonary and cardiac interstitial pathology. J Ultrasound 20(2):91-96. https://doi.org/10.1007/ s40477-017-0244-7

8. Sofia S (2013) Bedside US imaging in multiple trauma patients. Part 1: US findings and techniques. J Ultrasound 16(4):147-159. https://doi.org/10.1007/s40477-013-0047-4

9. Orso D, Ban A, Guglielmo N (2018) Lung ultrasound in diagnosing pneumonia in childhood: a systematic review and metaanalysis. J Ultrasound 21(3):183-195. https://doi.org/10.1007/ s40477-018-0306-5

10. Boccatonda A, Primomo G, Cocco G, D’Ardes D, Marinari S, Montanari M, Giostra F, Schiavone C (2020) Not all abolished lung slidings are pneumothorax: the case of a particular lung atelectasis. J Ultrasound. https://doi.org/10.1007/s40477-020-00427 $-0$

11. Sperandeo M, Frongillo E, De Cosmo S, Cipriani C (2018) Transthoracic ultrasound in children. J Ultrasound 21(4):355-356. https://doi.org/10.1007/s40477-018-0339-9

12. Sofia S, Angelini F, Cianci V, Copetti R, Farina R, Scuderi $M$ (2009) Diffusion and practice of ultrasound in emergency medicine departments in Italy. J Ultrasound 12(3):112-117. https ://doi.org/10.1016/j.jus.2009.06.004

13. Orso D, Guglielmo N, Copetti R (2018) Lung ultrasound in diagnosing pneumonia in the emergency department: a systematic review and meta-analysis. Eur J Emerg Med 25(5):312-321. https ://doi.org/10.1097/MEJ.0000000000000517

14. Testa A, Soldati G, Copetti R, Giannuzzi R, Portale G, GentiloniSilveri N (2012) Early recognition of the 2009 pandemic influenza A (H1N1) pneumonia by chest ultrasound. Crit Care 16(1):R30. https://doi.org/10.1186/cc11201

15. Poggiali E, Dacrema A, Bastoni D, Tinelli V, Demichele E, Mateo Ramos P, Marcianò T, Silva M, Vercelli A, Magnacavallo A (2020) Can lung US help critical care clinicians in the early diagnosis of novel coronavirus (COVID-19) Pneumonia? Radiology 13:200847. https://doi.org/10.1148/radiol.2020200847

16. Reissig A, Copetti R (2014) Lung ultrasound in communityacquired pneumonia and in interstitial lung diseases. Respiration 87(3):179-189. https://doi.org/10.1159/000357449

17. Boccatonda A, Liberatore I, Cocco G, D’Ardes D, Marinari S, Schiavone C (2020) From an abdominal ultrasound to a lung disease passing through the diaphragm: a case of idiopathic pulmonary fibrosis. J Ultrasound. https://doi.org/10.1007/s40477-02000445-y

18. Demi M, Prediletto R, Soldati G, Demi L (2020) Physical mechanisms providing clinical information from ultrasound lung images: hypotheses and early confirmations. IEEE Trans Ultrason Ferroelectr Freq Control 67(3):612-623. https://doi.org/10.1109/ TUFFC.2019.2949597

19. Soldati G, Demi M, Smargiassi A, Inchingolo R, Demi L (2019) The role of ultrasound lung artifacts in the diagnosis of respiratory diseases. Expert Rev Respir Med 13(2):163-172. https://doi. org/10.1080/17476348.2019.1565997

20. Zanforlin A, Tursi F, Marchetti G, Pellegrino GM, Vigo B, Smargiassi A et al (2020) Clinical use and barriers of thoracic ultrasound: a survey of italian pulmonologists. Respiration 99(2):171-176. https://doi.org/10.1159/000504632

21. Généreux V, Chassé M, Girard F, Massicotte N, ChartrandLefebvre C, Girard M (2020) Effects of positive end-expiratory pressure/recruitment manoeuvres compared with zero endexpiratory pressure on atelectasis during open gynaecological surgery as assessed by ultrasonography: a randomised controlled trial. Br J Anaesth 124(1):101-109. https://doi.org/10.1016/j. bja.2019.09.040

22. Boccatonda A, Decorato V, Cocco G, Marinari S, Schiavone C (2018) Ultrasound evaluation of diaphragmatic mobility in patients with idiopathic lung fibrosis: a pilot study. Multidiscip Respir Med 14(14):1. https://doi.org/10.1186/s40248-018-0159-y

23. Rea G, Sperandeo M, Di Serafino M et al (2019) Neonatal and pediatric thoracic ultrasonography. J Ultrasound 22:121-130. https://doi.org/10.1007/s40477-019-00357-6

Publisher's Note Springer Nature remains neutral with regard to jurisdictional claims in published maps and institutional affiliations. 